\title{
Weed Control with Sulfentrazone plus a Low Rate of Imazethapyr in White Bean
}

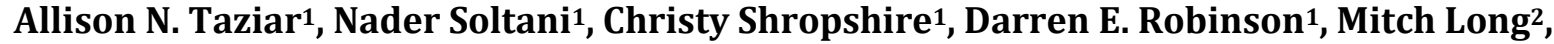 \\ Chris L. Gillard1, Peter H. Sikkema1 \\ ${ }^{1}$ University of Guelph Ridgetown Campus, Ridgetown, Canada \\ ${ }^{2}$ FMC Corporation, Saskatoon, Canada \\ Email: soltanin@uoguelph.ca
}

Received 9 June 2016; accepted 23 July 2016; published 26 July 2016

Copyright (C) 2016 by authors and Scientific Research Publishing Inc.

This work is licensed under the Creative Commons Attribution International License (CC BY).

http://creativecommons.org/licenses/by/4.0/

c) (i) Open Access

\begin{abstract}
Ontario dry bean (Phaseolus vulgaris L.) growers have few options for broadleaf weed control. Sulfentrazone is a group 14 herbicide that provides good control of several common Ontario weed species, and would provide another mode of action for broadleaf weed control if registered for use in Ontario dry beans. Five field studies were conducted during 2014 and 2015 to determine if a low dose of imazethapyr added to a tank mix of sulfentrazone + s-metolachlor would improve broadleaf control in white bean. Sulfentrazone (140 and 210 g.ai-ha-1) was mixed with imazethapyr and s-metolachlor and evaluated at 2 and 4 weeks after crop emergence for crop injury. Weed control was assessed visually at 4 and 8 weeks after herbicide application (WAA), and weed stand counts and biomass were determined at 8 WAA. Seed moisture and yield were determined at harvest. At 8 WAA, sulfentrazone (140 g.ai-ha-1) controlled pigweed species, common ragweed, common lambsquarters, wild mustard, barnyard grass and green foxtail $100 \%, 4 \%, 100 \%, 2 \%, 86 \%$ and $62 \%$, respectively. The addition of imazethapyr ( $37.5 \mathrm{~g} \cdot \mathrm{ai}^{\left.-\mathrm{ha}^{-1}\right)}$ to sulfentrazone $\left(140 \mathrm{~g} \cdot \mathrm{ai} \cdot \mathrm{ha}^{-1}\right) \mathrm{improved}$ the control of common ragweed, wild mustard and green foxtail by $19 \%, 98 \%$ and $33 \%$, respectively.

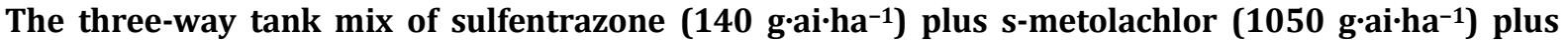
imazethapyr ( $37.5 \mathrm{~g} \cdot \mathrm{ai}^{\mathrm{h}} \mathrm{ha}^{-1}$ ) controlled pigweed species, common ragweed, common lambsquarters, wild mustard, barnyard grass and green foxtail $100 \%, 35 \%, 100 \%, 100 \%, 96 \%$ and $100 \%$, respectively. The tank mixes evaluated caused unacceptably high levels of crop injury; this study does not support the registration of sulfentrazone plus s-metolachlor + imazethapyr for use in Ontario white bean.
\end{abstract}

\section{Keywords}

Biomass, Density, Injury, Height, Navy Bean, Phaseolus vulgaris L. 


\section{Introduction}

In 2015, Ontario growers produced 115,000 tonnes of dry edible beans (Phaseolus vulgaris L.) on 52,600 ha of land [1]. White bean accounted for over half of this production, and has been the prominent market class of dry bean grown in Ontario for several decades [2]. Dry beans are extremely sensitive to weed pressure and do not compete well. Some studies have reported white bean yield losses between $68 \%$ and $77 \%$ due to weed interference [3]-[5]. Weeds can also reduce the quality of the beans through dockage, pick, or plant juices causing discolouration of the seed coat. Therefore, weed control is a critical component of dry bean production. One method of controlling weeds is with the use of herbicides.

Sulfentrazone is a group 14, protoporphyrinogen oxidase IX (PPO) inhibitor herbicide in the aryl triazone family, recently registered in Ontario for use in soybean (Glycine max L.). Sulfentrazone causes excessive accumulation of protoporphyrinogen IX in the chloroplasts of plant cells. The excess protoporphyrinogen IX leaks out of the chloroplast and undergoes various reactions in the cytoplasm to produce $\mathrm{O}^{+}$radicals, which then peroxidizes lipids in cell membranes. Cell membrane integrity is compromised, the cell dies, and the plant can no longer function normally [1]. Sulfentrazone is primarily a broadleaf herbicide that also has some grass activity, and controls common Ontario weed species such as redroot pigweed (Amaranthus retroflexus L.), common waterhemp (Amaranthus tuberculatus VAR. rudis), common lambsquarters (Chenopodium album L.), crabgrass (Digitaria), foxtail (Setaria) and Panicum species [6]-[8].

Imazethapyr is a group 2, imadazolinone herbicide that provides broadleaf and grass control [9]. In Ontario, it is registered for soil and post-emergence (POST) application in soybean, pre-emergence (PRE) application in lima bean (Phaseolus lunatus L.), and pre-plant incorporated (PPI) and PRE application in peas (Pisum savitum L.), snow peas (Pisum sativum L. var. saccharatum), adzuki bean (Vigna angularis L.) and all Phaseolus vulgaris market classes [10]. Imazethapyr controls green foxtail (Setaria viridis (L.) Beauv.), yellow foxtail (Setaria glauca (L.) Beauv.), proso millet (Panicum miliaceum L.), lambsquarters, wild mustard (Sinapis arvensis L.), annual nightshades (Solanum spp.), pigweeds (Amaranthus spp.), and velvetleaf (Abutilon theophrasti Medik.) [10]. Studies have shown that imazethapyr applied PRE can provide effective weed control at reduced rates [11] [12] (Sikkema et al. 2005; Soltani et al. 2007).

S-metolachlor is a group 15, chloroacetamide herbicide that controls eastern black nightshade (Solanum ptycanthum Dunal), barnyard grass (Echinochloa crusgalli (L.) Beauv.), crabgrass, fall panicum (Panicum dichotomiflorum (L.) Michx.), witchgrass (Panicum capillare L.), and foxtails [10]. It is the only grass herbicide currently registered for PRE application in Ontario dry beans.

There is little information on sulfentrazone for broadleaf weed control in Ontario. If registered in Ontario, sulfentrazone would provide dry bean growers with a different mode of action for broadleaf control. The objectives of this research were to determine if a low rate of imazethapyr added to sulfentrazone would improve broadleaf weed control, and to determine the tolerance of white bean to sulfentrazone, s-metolachlor, and imazethapyr applied alone and in combination.

\section{Materials and Methods}

Five field studies were conducted over 2014 and 2015 at the Huron Research Station (Exeter) and the University of Guelph Ridgetown Campus (Ridgetown) in Ontario, Canada. The soil at the 2014 site near Exeter was a clay loam comprised of $31 \%, 42 \%$ and $27 \%$ sand, silt, and clay, respectively, and had a $\mathrm{pH}$ of 7.8 and organic matter content of $4.3 \%$. In 2015, the first Exeter site was a loamy soil of 32\% sand, $42 \%$ silt, $26 \%$ clay, pH of 7.7 and organic matter content of $3.2 \%$. The second Exeter site in 2015 was also a loam consisting of $35 \%$ sand, $43 \%$ silt and 22\% clay, pH of 7.6 and organic matter content of 3.6\%. In 2015, Ridgetown soils were sandy clay loams, with the first site having a sand, silt and clay content of $52 \%, 24 \%$ and $24 \%$, respectively, a pH of 7.3 and organic matter content of $4.3 \%$, and the second site consisting of $46 \%$ sand, $27 \%$ silt, $27 \%$ clay, a pH of 6.4 and organic matter content of $3.7 \%$. All sites were prepared by moldboard ploughing in the fall and two passes with an s-tine cultivator with rolling baskets in the spring. Plots were $3 \mathrm{~m}$ by $10 \mathrm{~m}$ in Exeter and $3 \mathrm{~m}$ by $8 \mathrm{~m}$ in Ridgetown, and were seeded with white bean variety T9905 at a rate of approximately 233,000 seeds ha ${ }^{-1}, 4$ to 5 $\mathrm{cm}$ deep in rows spaced $75 \mathrm{~cm}$ apart. All experiments were seeded in late May to early June.

The experiments were arranged in randomized complete block design with four replicates of thirteen treatments. An untreated weedy control and a weed-free control were included in each replicate; the weed-free control was sprayed with s-metolachlor (1050 g·ai ha $\left.{ }^{-1}\right)+$ halosulfuron (35 g.ai ha $\left.{ }^{-1}\right)$ applied PRE and maintained 
weed-free by hand-hoeing for the rest of the season. Herbicide treatments included s-metolachlor $\left(1050 \mathrm{~g} \cdot \mathrm{ai}^{-h^{-1}}{ }^{-1}\right)$ for grass control, sulfentrazone at 140 and $210 \mathrm{~g} \cdot \mathrm{ai} \cdot \mathrm{ha}^{-1}$, imazethapyr at $37.5 \mathrm{~g} \cdot \mathrm{ai} \cdot \mathrm{ha}^{-1}$ (half the registered label rate), and various co-applications of these herbicides (Table 1). Herbicides were applied PRE using a $1.5 \mathrm{~m}$ handheld boom with four ULD 120-02 nozzles (Hypro, New Brighton, MN) attached to a $\mathrm{CO}_{2}$ pressurized backpack sprayer calibrated to deliver $200 \mathrm{~L} \cdot \mathrm{ha}^{-1}$ at $240 \mathrm{kPa}$.

White bean injury was assessed at two and four weeks after crop emergence (WAE) by visually comparing the white beans in each herbicide treatment to the weed-free control, and was ranked from $0 \%$ (no injury) to $100 \%$ (total plant death). Weed control was assessed by species at four and eight weeks after herbicide application (WAA) by visually comparing the weeds in each herbicide treatment to the weedy control, and was ranked from $0 \%$ (no control) to $100 \%$ (complete control). At 8 WAA, weed density and biomass for each species were determined by a stand count in $1 \mathrm{~m}^{2}$ per plot, after which the weeds were cut at the soil surface, placed in a separate paper bag for each species, dried in a kiln, and weighed. Seed moisture and yield—adjusted to $18 \%$ moisture-were recorded at harvest.

All analyses were performed using SAS 9.4 software (SAS Institute Inc., NC). Data were partitioned into the fixed effects of treatment and the random effects of replicate, environment (year-location combination), replicate nested within environment, and environment by treatment interaction. The F-test was used to determine the significance of the fixed effects and the Z-test was used to determine the significance of the random effects. The UNIVARIATE procedure was used to test the assumptions of homogeneity and normality of the residuals with various transformations of the data. All transformations applied to the data met the assumptions, therefore the transformation that produced the least amount of error was selected for the analysis. Crop injury, seed moisture, yield, and weed control data were transformed with the arcsine square root transformation. Weed density and biomass data were analysed with a logarithmic transformation. The MIXED procedure was used to perform an analysis of variance on all data, using a Fisher's Protected LSD test with $\alpha=0.05$. Data were converted back to the original scale for presentation.

Table 1. Mean visible injury, seed moisture content at harvest, and yield (adjusted to 18\% moisture) of white bean treated with sulfentrazone, imazethapyr and s-metolachlor PRE in five field studies over two years at the University of Guelph Ridgetown Campus, Ridgetown, ON (2015) and Huron Research Station, Exeter, ON (2014, 2015) .

\begin{tabular}{|c|c|c|c|c|c|c|c|c|}
\hline \multirow{3}{*}{ Treatment } & \multirow[t]{2}{*}{ Rate } & \multicolumn{3}{|c|}{ Dry bean injury } & \multicolumn{2}{|c|}{ Seed moisture content } & \multirow{2}{*}{\multicolumn{2}{|c|}{ Yield }} \\
\hline & & \multicolumn{3}{|c|}{$2 \mathrm{WAE}$} & \multicolumn{2}{|c|}{$4 \mathrm{WAE}$} & & \\
\hline & \multirow[t]{2}{*}{$\mathrm{g} \cdot \mathrm{ai} \cdot \mathrm{ha}^{-1}$} & \multicolumn{5}{|c|}{$\%$} & \multicolumn{2}{|c|}{$\mathrm{T} \cdot \mathrm{ha}^{-1}$} \\
\hline Untreated Control & & 0 & $\mathrm{a}$ & 0 & $\mathrm{a}$ & $17.8 \mathrm{a}$ & 1.20 & $\mathrm{C}$ \\
\hline \multicolumn{2}{|l|}{ Weed Free Control } & 0 & $\mathrm{a}$ & 0 & a & $17.2 \mathrm{a}$ & 2.52 & a \\
\hline S-metolachlor & 1050 & 3 & abc & 3 & abc & 17.9 a & 1.68 & bc \\
\hline Sulfentrazone & 140 & 5 & abc & 3 & abcd & $17.7 \mathrm{a}$ & 1.42 & bc \\
\hline Sulfentrazone & 210 & 12 & cde & 12 & cdef & $17.9 \mathrm{a}$ & 1.49 & bc \\
\hline Imazethapyr & 37.5 & 2 & $\mathrm{ab}$ & 2 & $\mathrm{ab}$ & $17.5 \mathrm{a}$ & 1.67 & bc \\
\hline Sulfentrazone + s-metolachlor & $140+1050$ & 13 & cde & 14 & defg & $17.4 \mathrm{a}$ & 1.60 & bc \\
\hline Sulfentrazone + s-metolachlor & $210+1050$ & 23 & ef & 22 & fg & $17.8 \mathrm{a}$ & 1.61 & bc \\
\hline Imazethapyr + s-metolachlor & $37.5+1050$ & 6 & abcd & 6 & abcde & $17.7 \mathrm{a}$ & 1.71 & bc \\
\hline Sulfentrazone + imazethapyr & $140+37.5$ & 6 & abcd & 7 & abcde & 17.9 a & 1.80 & abc \\
\hline Sulfentrazone + imazethapyr & $210+37.5$ & 17 & def & 18 & egf & 17.9 a & 1.67 & bc \\
\hline $\begin{array}{c}\text { Sulfentrazone + s-metolachlor + } \\
\text { imazethapyr }\end{array}$ & $140+1050+37.5$ & 11 & bcde & 10 & bcdef & $17.6 \mathrm{a}$ & 1.89 & $\mathrm{ab}$ \\
\hline $\begin{array}{c}\text { Sulfentrazone + s-metolachlor + } \\
\text { imazethapyr }\end{array}$ & $210+1050+37.5$ & 31 & $\mathrm{f}$ & 30 & g & $17.8 \mathrm{a}$ & 1.60 & bc \\
\hline $\mathrm{SE}( \pm)$ & & & & & & 0.02 & & \\
\hline
\end{tabular}

a Abbreviations: PRE, pre-emergence; WAE, weeks after emergence. Data are averaged for years and locations. Means followed by the same letter within a column are not statistically different at $\alpha=0.05$ using Fisher's Protected LSD. 


\section{Results and Discussion}

Analyses determined that replicate, environment, and replicate within environment effects were not significant; therefore data were pooled for analysis.

\subsection{Crop Injury}

White bean injury symptoms included leaf malformation and stunting. S-metolachlor, imazethapyr, and imazethapyr + s-metolachlor caused 3\%, 2\%, and 6\% injury, respectively, at 2 and 4 WAE (Table 1). Sulfentrazone $\left(140 \mathrm{~g} \cdot \mathrm{ai} \cdot \mathrm{ha}^{-1}\right)$ caused $5 \%$ injury at $2 \mathrm{WAE}$ and $3 \%$ injury at $4 \mathrm{WAE}$, while sulfentrazone $\left(210 \mathrm{~g} \cdot \mathrm{ai} \cdot \mathrm{ha}^{-1}\right)$ caused $12 \%$ injury at 2 and 4 WAE. Sulfentrazone (140 and 210 g-ai $\left.\cdot \mathrm{ha}^{-1}\right)+$ s-metolachlor caused up to $23 \%$ at $2 \mathrm{WAE}$, and up to $22 \%$ at $4 \mathrm{WAE}$. The levels of injury caused by sulfentrazone + s-metolachlor were not different than the injury produced by sulfentrazone alone, but were greater than the weed-free control. Sulfentrazone $\left(140 \mathrm{~g} \cdot \mathrm{ai} \cdot \mathrm{ha}^{-1}\right)+$ imazethapyr caused up to $7 \%$ injury and sulfentrazone $\left(210 \mathrm{~g} \cdot a \mathrm{i} \cdot \mathrm{ha}^{-1}\right)+$ imazethapyr caused up to $18 \%$ injury 4 WAE. This is consistent with a study by Soltani et al. [13], which found that sulfentrazone $\left(140 \mathrm{~g} \cdot \mathrm{ai}^{\mathrm{h}} \mathrm{ha}^{-1}\right)+$ imazethapyr (37.5 g.ai $\left.\cdot \mathrm{ha}^{-1}\right)$ caused $6 \%$ injury and sulfentrazone $\left(280 \mathrm{~g} \cdot \mathrm{ai} \cdot \mathrm{ha}^{-1}\right)+$ imazethapyr $\left(37.5 \mathrm{~g} \cdot \mathrm{ai} \cdot \mathrm{ha}^{-1}\right)$ caused $19 \%$ injury in white bean. Sulfentrazone $\left(140 \mathrm{~g} \cdot \mathrm{ai} \cdot \mathrm{ha}^{-1}\right)+\mathrm{s}$-metolachlor + imazethapyr caused $11 \%$ injury at $2 \mathrm{WAE}$ and $10 \%$ injury at $4 \mathrm{WAE}$. In contrast, sulfentrazone $\left(210 \mathrm{~g} \cdot \mathrm{ai} \cdot \mathrm{ha} \mathrm{H}^{-1}\right)+\mathrm{s}$-metolachlor + imazethapyr produced the highest injury of all the treatments, causing 31\% injury at 2 WAE and $30 \%$ injury at 4 WAE. As imazethapyr + s-metolachlor only produced $6 \%$ injury, these high levels of injury are likely due to the addition of the high rate of sulfentrazone to the tank mix.

\subsection{Weed Control, Density and Biomass}

\subsubsection{Pigweed Species}

Green pigweed (Amaranthus powelli L.) was the dominant pigweed species at the Ridgetown locations and redroot pigweed (A. retroflexus) was the dominant species at the Exeter locations. Pigweed species were combined for analysis. At 4 WAA, all herbicides and herbicide combinations provided excellent ( $\geq 92 \%)$ control of pigweeds (Table 2). Sulfentrazone (140 and $210 \mathrm{~g} \cdot \mathrm{ai}^{-\mathrm{ha}^{-1}}$ ) controlled pigweeds $100 \%$, while s-metolachlor provided 93\% control and imazethapyr provided 92\% control. There was no improvement in pigweed control when sulfentrazone was used in a co-application versus sulfentrazone applied alone. At 8 WAA, sulfentrazone, s-metolachlor and imazethapyr controlled pigweed species $100 \%, 79 \%$ and $87 \%$, respectively. Tank mixes of sulfentrazone + s-metolachlor, sulfentrazone + imazethapyr and sulfentrazone + s-metolachlor + imazethapyr provided excellent ( $\geq 98 \%$ ) control of pigweeds. All herbicides and herbicide combinations reduced pigweed density and biomass by $91 \%$ to $100 \%$ and $86 \%$ to $100 \%$, respectively, and were not different from the weed-free control. This is consistent with other studies which found excellent control of redroot pigweed with sulfentrazone at similar rates [14] [15].

\subsubsection{Common Ragweed}

At 4 WAA, sulfentrazone alone at 140 and $210 \mathrm{~g} \cdot \mathrm{ai} \cdot \mathrm{ha}^{-1}$ provided $16 \%$ and $23 \%$ control of common ragweed, respectively (Table 3). S-metolachlor and imazethapyr provided 28\% and 37\% control, respectively. There was no improvement in control when sulfentrazone (140 or $210 \mathrm{~g} \cdot \mathrm{ai} \cdot \mathrm{ha}^{-1}$ ) was co-applied with imazethapyr, s-metolachlor or s-metolachlor + imazethapyr. Niekamp et al. [7] noted improved control of common ragweed when sulfentrazone was combined with the imidazolinone herbicide imazaquin, though the rates used in that study were much higher than in the present study. Sulfentrazone $\left(140\right.$ or $\left.210 \mathrm{~g} \cdot \mathrm{ai} \cdot \mathrm{ha}^{-1}\right)+\mathrm{s}$-metolachlor + imazethapyr improved ragweed control to $74 \%$ and $61 \%$, respectively, but the level of control was not different from co-applications of sulfentrazone (140 and $\left.210 \mathrm{~g} \cdot \mathrm{ai}^{\mathrm{h}} \mathrm{ha}^{-1}\right)+$ imazethapyr or sulfentrazone (140 and $\left.210 \mathrm{~g} \cdot \mathrm{ai} \cdot \mathrm{ha} \mathrm{a}^{-1}\right)+\mathrm{s}$-metolachlor. Sulfentrazone $\left(140 \mathrm{~g} \cdot \mathrm{ai}^{\mathrm{i}} \mathrm{ha}^{-1}\right)+\mathrm{s}$-metolachlor + imazethapyr provided the highest level of control at $74 \%$, which was not statistically different from the weed-free control. At 8 WAA, control decreased for all herbicides and herbicide combinations. Sulfentrazone (140 and $210 \mathrm{~g} \cdot \mathrm{ai}^{\mathrm{h}} \mathrm{ha}^{-1}$ ) provided $4 \%$ and $10 \%$ control of common ragweed, respectively, sulfentrazone + s-metolachlor provided up to $18 \%$ control and sulfentrazone + imazethapyr provided up to $23 \%$ control. Sulfentrazone (140 and $\left.210 \mathrm{~g} \cdot \mathrm{ai}^{\mathrm{h}} \mathrm{ha}^{-1}\right)+\mathrm{s}$-metolachlor + imazethapyr provided 35\% and 39\% control, respectively. Ragweed density and biomass were reduced up to $46 \%$ and $26 \%$, respectively, but were not reduced compared to the weedy control. 
Table 2. Mean control, plant density and biomass ratings for pigweeds (A. powelli and A. retroflexus) treated with tank mixes of sulfentrazone, imazethapyr and s-metolachlor applied PRE in Ridgetown, ON and Exeter, ON, Canada over two years $(2014,2015)^{\mathrm{a}}$.

\begin{tabular}{|c|c|c|c|c|c|c|c|c|c|}
\hline \multirow{3}{*}{ Treatment } & Rate & \multicolumn{4}{|c|}{ Control } & \multicolumn{2}{|c|}{ Density 8 WAA } & \multicolumn{2}{|c|}{ Biomass 8 WAA } \\
\hline & & \multicolumn{2}{|c|}{4 WAA } & \multicolumn{2}{|c|}{8 WAA } & & & & \\
\hline & \multirow[t]{2}{*}{ g.ai $\cdot h a^{-1}$} & \multicolumn{4}{|c|}{$\%$} & \multicolumn{2}{|c|}{ plants $\mathrm{m}^{-2}$} & \multicolumn{2}{|c|}{$\mathrm{g} \cdot \mathrm{m}^{-2}$} \\
\hline Untreated Control & & 0 & c & 0 & c & 8.5 & $\mathrm{~b}$ & 6.3 & $\mathrm{~b}$ \\
\hline Weed Free Control & & 100 & a & 100 & a & 0.0 & a & 0.0 & a \\
\hline S-metolachlor & 1050 & 93 & $\mathrm{ab}$ & 79 & $\mathrm{~b}$ & 0.8 & a & 0.9 & a \\
\hline Sulfentrazone & 140 & 100 & $a b$ & 100 & a & 0.2 & a & 0.1 & a \\
\hline Sulfentrazone & 210 & 100 & $\mathrm{ab}$ & 100 & a & 0.1 & $\mathrm{a}$ & 0.0 & a \\
\hline Imazethapyr & 37.5 & 92 & $\mathrm{~b}$ & 87 & $\mathrm{ab}$ & 0.4 & $\mathrm{a}$ & 0.3 & a \\
\hline Sulfentrazone + s-metolachlor & $140+1050$ & 99 & $\mathrm{ab}$ & 98 & $\mathrm{ab}$ & 0.1 & a & 0.0 & a \\
\hline Sulfentrazone + s-metolachlor & $210+1050$ & 100 & a & 100 & a & 0.0 & a & 0.0 & a \\
\hline Imazethapyr + s-metolachlor & $37.5+1050$ & 100 & $\mathrm{ab}$ & 99 & $\mathrm{ab}$ & 0.2 & $\mathrm{a}$ & 0.2 & a \\
\hline Sulfentrazone + imazethapyr & $140+37.5$ & 100 & $\mathrm{ab}$ & 100 & a & 0.0 & a & 0.0 & a \\
\hline Sulfentrazone + imazethapyr & $210+37.5$ & 100 & $\mathrm{ab}$ & 100 & a & 0.0 & a & 0.0 & a \\
\hline $\begin{array}{l}\text { Sulfentrazone }+ \text { s-metolachlor }+ \\
\text { imazethapyr }\end{array}$ & $140+1050+37.5$ & 100 & $a b$ & 100 & a & 0.0 & a & 0.0 & a \\
\hline $\begin{array}{l}\text { Sulfentrazone }+ \text { s-metolachlor }+ \\
\text { imazethapyr }\end{array}$ & $210+1050+37.5$ & 100 & a & 100 & a & 0.0 & a & 0.0 & $\mathrm{a}$ \\
\hline $\mathrm{SE}( \pm)$ & & \multicolumn{2}{|c|}{0.06} & \multicolumn{2}{|c|}{0.08} & \multicolumn{2}{|c|}{0.15} & \multicolumn{2}{|c|}{0.18} \\
\hline
\end{tabular}

${ }^{a}$ Abbreviations: PRE, pre-emergence; WAA, weeks after application. Means followed by the same letter within a column are not statistically different at $\alpha=0.05$ using Fisher's Protected LSD. Data are averaged for years and locations.

Table 3. Mean control, plant density and biomass ratings for common ragweed (Ambrosia artemisiifolia) treated with tank mixes of sulfentrazone, imazethapyr and s-metolachlor applied PRE in Ridgetown, ON and Exeter, ON, Canada over two years $(2014,2015)^{\mathrm{a}}$.

\begin{tabular}{|c|c|c|c|c|c|c|c|c|c|}
\hline \multirow{4}{*}{$\begin{array}{c}\text { Treatment } \\
\text { Untreated Control }\end{array}$} & \multirow{4}{*}{$\begin{array}{c}\text { Rate } \\
\mathrm{g} \cdot \mathrm{ai} \cdot \mathrm{ha}^{-1}\end{array}$} & \multicolumn{4}{|c|}{ Control } & \multicolumn{2}{|c|}{ Density 8 WAA } & \multicolumn{2}{|c|}{ Biomass 8 WAA } \\
\hline & & \multicolumn{2}{|c|}{4 WAA } & \multicolumn{2}{|c|}{8 WAA } & & & & \\
\hline & & \multicolumn{4}{|c|}{$\%$} & \multicolumn{2}{|c|}{ plants $\mathrm{m}^{-2}$} & \multicolumn{2}{|c|}{$g \cdot m^{-2}$} \\
\hline & & 0 & c & 0 & c & 7.6 & $\mathrm{~b}$ & 12.5 & $\mathrm{~b}$ \\
\hline Weed Free Control & & 100 & a & 100 & a & 0.1 & a & 0.1 & a \\
\hline S-metolachlor & 1050 & 28 & bc & 1 & bc & 5.0 & $\mathrm{~b}$ & 12.7 & $\mathrm{~b}$ \\
\hline Sulfentrazone & 140 & 16 & bc & 4 & bc & 4.1 & $\mathrm{~b}$ & 9.2 & $\mathrm{~b}$ \\
\hline Sulfentrazone & 210 & 23 & bc & 10 & bc & 5.2 & $\mathrm{~b}$ & 15.3 & $\mathrm{~b}$ \\
\hline Imazethapyr & 37.5 & 37 & $\mathrm{~b}$ & 23 & bc & 7.0 & $\mathrm{~b}$ & 12.8 & $\mathrm{~b}$ \\
\hline Sulfentrazone + s-metolachlor & $140+1050$ & 33 & bc & 18 & bc & 4.8 & $\mathrm{~b}$ & 14.5 & $\mathrm{~b}$ \\
\hline Sulfentrazone + s-metolachlor & $210+1050$ & 56 & $\mathrm{~b}$ & 17 & bc & 4.6 & $\mathrm{~b}$ & 14.9 & $\mathrm{~b}$ \\
\hline Imazethapyr + s-metolachlor & $37.5+1050$ & 36 & $\mathrm{~b}$ & 29 & bc & 4.8 & $\mathrm{~b}$ & 10.8 & $\mathrm{~b}$ \\
\hline Sulfentrazone + imazethapyr & $140+37.5$ & 41 & $\mathrm{~b}$ & 23 & bc & 6.3 & $\mathrm{~b}$ & 15.3 & $\mathrm{~b}$ \\
\hline Sulfentrazone + imazethapyr & $210+37.5$ & 45 & $\mathrm{~b}$ & 20 & bc & 5.1 & $\mathrm{~b}$ & 13.3 & $\mathrm{~b}$ \\
\hline $\begin{array}{c}\text { Sulfentrazone }+ \text { s-metolachlor }+ \\
\text { imazethapyr }\end{array}$ & $140+1050+37.5$ & 74 & $\mathrm{ab}$ & 35 & $\mathrm{~b}$ & 4.1 & $\mathrm{~b}$ & 9.3 & $\mathrm{~b}$ \\
\hline $\begin{array}{l}\text { Sulfentrazone + s-metolachlor }+ \\
\text { imazethapyr }\end{array}$ & $210+1050+37.5$ & 61 & $\mathrm{~b}$ & 39 & $\mathrm{~b}$ & 4.4 & $\mathrm{~b}$ & 10.7 & $\mathrm{~b}$ \\
\hline SE $( \pm)$ & & & & & & & & & \\
\hline
\end{tabular}

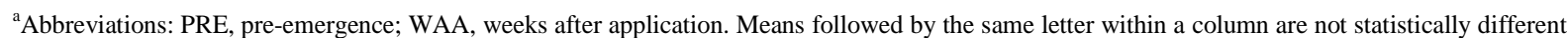
at $\alpha=0.05$ using Fisher's Protected LSD. Data are averaged for years and locations. 


\subsubsection{Common Lambsquarters}

At 4 and 8 WAA, sulfentrazone (140 and $210 \mathrm{~g} \cdot a \mathrm{i} \cdot \mathrm{ha}^{-1}$ ) provided $100 \%$ control of common lambsquarters (Table 4). S-metolachlor provided 27\% control at both 4 and 8 WAA, while imazethapyr provided $99 \%$ control at 4 WAA and $97 \%$ control at 8 WAA. Sulfentrazone $\left(140\right.$ and $\left.210 \mathrm{~g} \cdot a \cdot \cdot \mathrm{ha}^{-1}\right)+$ s-metolachlor + imazethapyr provided $100 \%$ control of lambsquarters at 4 and 8 WAA. Sulfentrazone (140 and $\left.210 \mathrm{~g} \cdot \mathrm{ai}^{\mathrm{i}} \mathrm{ha}^{-1}\right)$ alone reduced lambsquarters density by $99 \%$ and biomass by $100 \%$. Sulfentrazone $\left(140 \mathrm{~g} \cdot \mathrm{ai} \cdot \mathrm{ha} \mathrm{C}^{-1}\right)+\mathrm{s}$-metolachlor + imazethapyr reduced both density and biomass by $100 \%$, while sulfentrazone $\left(210 \mathrm{~g} \cdot \mathrm{ai}^{-\mathrm{h}} \mathrm{a}^{-1}\right)+\mathrm{s}$-metolachlor + imazethapyr reduced lambsquarters density and biomass by $98 \%$. All tank mixes reduced density and biomass relative to the weedy control. Other studies have reported excellent lambsquarters control when sulfentrazone was applied alone or in a co-application [16] [17].

\subsubsection{Wild Mustard}

At 4 WAA, sulfentrazone at 140 and $210 \mathrm{~g} \cdot a \mathrm{a} \cdot \mathrm{ha}^{-1}$ provided $35 \%$ and $80 \%$ control, respectively, s-metolachlor provided 31\% control, and imazethapyr provided 97\% control of wild mustard (Table 5). Sulfentrazone (140 g.ai $\cdot \mathrm{ha}^{-1}$ ) tank-mixed with s-metolachlor improved control to $76 \%$, while sulfentrazone $\left(210 \mathrm{~g} \cdot \mathrm{ai}^{\mathrm{h}} \mathrm{ha}^{-1}\right)+\mathrm{s}$-metolachlor did not provide improved control compared to sulfentrazone alone. The level of control with sulfentrazone (140 and $\left.210 \mathrm{~g} \cdot \mathrm{ai} \cdot \mathrm{ha} \mathrm{a}^{-1}\right)+\mathrm{s}$-metolachlor decreased as the season progressed, with only $8 \%$ and $25 \%$ control, respectively, at 8 WAA. In contrast, sulfentrazone (140 and $210 \mathrm{~g} \cdot \mathrm{ai}^{-h^{-1}}{ }^{-1}$ ) plus imazethapyr provided $97 \%$ to $100 \%$ control throughout the season. The co-application of all three herbicides provided $100 \%$ control at both 4 and 8 WAA. Sikkema et al. [11] found that imazethapyr applied PRE at rates as low as $15 \mathrm{~g} \cdot \mathrm{ai}^{\mathrm{h}} \mathrm{ha}^{-1}$ still provided up to $100 \%$ control of wild mustard, which likely contributes to the high level of control throughout the season. Sulfentrazone alone at 140 and $210 \mathrm{~g} \cdot \mathrm{ai} \cdot \mathrm{ha}^{-1}$ reduced wild mustard density by $7 \%$ and $55 \%$, respectively, but was not an improvement compared to the weedy control. There was no reduction in density when sulfentrazone was combined with s-metolachlor, but sulfentrazone (140 and $\left.210 \mathrm{~g} \cdot \mathrm{ai} \cdot \mathrm{ha}^{-1}\right)+$ imazethapyr reduced density by up to $97 \%$. Similarly, sulfentrazone alone reduced wild mustard biomass by up to $33 \%$ but was not different

Table 4. Mean control, plant density and biomass ratings for common lamb’s quarters (Chenopodium album) treated with tank mixes of sulfentrazone, s-metolachlor and imazethapyr applied PRE in Ridgetown, ON and Exeter, ON, Canada over two years $(2014,2015)^{\mathrm{a}}$.

\begin{tabular}{|c|c|c|c|c|c|c|c|c|c|}
\hline \multirow{3}{*}{ Treatment } & Rate & \multicolumn{4}{|c|}{ Control } & \multicolumn{2}{|c|}{ Density 8 WAA } & \multicolumn{2}{|c|}{ Biomass 8 WAA } \\
\hline & & \multicolumn{2}{|c|}{4 WAA } & \multicolumn{2}{|c|}{8 WAA } & & & & \\
\hline & \multirow[t]{2}{*}{$\mathrm{g} \cdot \mathrm{ai} \cdot \mathrm{ha}{ }^{-1}$} & \multicolumn{4}{|c|}{ - \% } & \multicolumn{2}{|c|}{ plants $\mathrm{m}^{-2}$} & \multicolumn{2}{|c|}{$\mathrm{g} \cdot \mathrm{m}^{-2}$} \\
\hline Untreated Control & & 0 & c & 0 & c & 11.8 & c & 5.5 & $\mathrm{~b}$ \\
\hline Weed Free Control & & 100 & a & 100 & a & 0.0 & a & 0.0 & a \\
\hline S-metolachlor & 1050 & 27 & $\mathrm{~b}$ & 27 & $\mathrm{~b}$ & 4.6 & $\mathrm{~b}$ & 4.8 & $\mathrm{~b}$ \\
\hline Sulfentrazone & 140 & 100 & $\mathrm{a}$ & 100 & $\mathrm{a}$ & 0.1 & $\mathrm{a}$ & 0.0 & $\mathrm{a}$ \\
\hline Sulfentrazone & 210 & 100 & $\mathrm{a}$ & 100 & $\mathrm{a}$ & 0.1 & $\mathrm{a}$ & 0.0 & $\mathrm{a}$ \\
\hline Imazethapyr & 37.5 & 99 & $\mathrm{a}$ & 97 & $\mathrm{a}$ & 2.3 & $\mathrm{~b}$ & 0.1 & $\mathrm{a}$ \\
\hline Sulfentrazone + s-metolachlor & $140+1050$ & 100 & $\mathrm{a}$ & 100 & $\mathrm{a}$ & 0.0 & $\mathrm{a}$ & 0.0 & $\mathrm{a}$ \\
\hline Sulfentrazone + s-metolachlor & $210+1050$ & 100 & a & 100 & $\mathrm{a}$ & 0.0 & $\mathrm{a}$ & 0.0 & $\mathrm{a}$ \\
\hline Imazethapyr + s-metolachlor & $37.5+1050$ & 99 & $\mathrm{a}$ & 95 & $\mathrm{a}$ & 0.6 & $\mathrm{a}$ & 0.1 & $\mathrm{a}$ \\
\hline Sulfentrazone + imazethapyr & $140+37.5$ & 100 & $\mathrm{a}$ & 100 & $\mathrm{a}$ & 0.0 & $\mathrm{a}$ & 0.0 & $\mathrm{a}$ \\
\hline Sulfentrazone + imazethapyr & $210+37.5$ & 99 & $\mathrm{a}$ & 100 & $\mathrm{a}$ & 0.0 & $\mathrm{a}$ & 0.0 & $\mathrm{a}$ \\
\hline $\begin{array}{c}\text { Sulfentrazone }+ \text { s-metolachlor }+ \\
\text { imazethapyr }\end{array}$ & $140+1050+37.5$ & 100 & $\mathrm{a}$ & 100 & $\mathrm{a}$ & 0.0 & $\mathrm{a}$ & 0.0 & $\mathrm{a}$ \\
\hline $\begin{array}{c}\text { Sulfentrazone + s-metolachlor }+ \\
\text { imazethapyr }\end{array}$ & $210+1050+37.5$ & 100 & $\mathrm{a}$ & 100 & $\mathrm{a}$ & 0.2 & $\mathrm{a}$ & 0.1 & $\mathrm{a}$ \\
\hline $\mathrm{SE}( \pm)$ & & \multicolumn{2}{|c|}{0.08} & \multicolumn{2}{|c|}{0.09} & \multicolumn{2}{|c|}{0.16} & \multicolumn{2}{|c|}{0.13} \\
\hline
\end{tabular}

${ }^{a}$ Abbreviations: PRE, pre-emergence; WAA, weeks after application. Means followed by the same letter within a column are not statistically different at $\alpha=0.05$ using Fisher's Protected LSD. Data are averaged for years and locations. 
Table 5. Mean control, plant density and biomass ratings for wild mustard (Sinapis arvensis) treated with tank mixes of sulfentrazone, imazethapyr and s-metolachlor applied PRE in Exeter, ON, Canada over two years (2014, 2015) ${ }^{\mathrm{a}}$.

\begin{tabular}{|c|c|c|c|c|c|c|c|c|c|}
\hline \multirow{3}{*}{ Treatment } & \multirow[t]{2}{*}{ Rate } & \multicolumn{4}{|c|}{ Control } & \multicolumn{2}{|c|}{ Density 8 WAA } & \multicolumn{2}{|c|}{ Biomass 8 WAA } \\
\hline & & \multicolumn{2}{|c|}{4 WAA } & \multicolumn{2}{|c|}{8 WAA } & & & & \\
\hline & \multirow[t]{2}{*}{$\mathrm{g} \cdot \mathrm{ai} \cdot \mathrm{ha}^{-1}$} & \multicolumn{4}{|c|}{ - \% } & \multicolumn{2}{|c|}{ plants $\mathrm{m}^{-2}$} & \multicolumn{2}{|c|}{$\mathrm{g} \cdot \mathrm{m}^{-2}$} \\
\hline Untreated Control & & 0 & $\mathrm{f}$ & 0 & c & 31.8 & $\mathrm{e}$ & 98.5 & c \\
\hline Weed Free Control & & 100 & a & 100 & $\mathrm{a}$ & 0.0 & a & 0.0 & a \\
\hline S-metolachlor & 1050 & 31 & e & 0 & c & 26.4 & e & 114.2 & c \\
\hline Sulfentrazone & 140 & 35 & e & 2 & c & 29.7 & e & 80.2 & c \\
\hline Sulfentrazone & 210 & 80 & $\mathrm{~cd}$ & 17 & bc & 14.4 & de & 66.1 & bc \\
\hline Imazethapyr & 37.5 & 97 & abcd & 99 & a & 0.6 & $\mathrm{ab}$ & 0.0 & a \\
\hline Sulfentrazone + s-metolachlor & $140+1050$ & 76 & d & 8 & c & 10.2 & cde & 33.7 & bc \\
\hline Sulfentrazone + s-metolachlor & $210+1050$ & 83 & bcd & 25 & bc & 6.7 & bcde & 31.5 & bc \\
\hline Imazethapyr + s-metolachlor & $37.5+1050$ & 91 & acd & 76 & $\mathrm{ab}$ & 2.8 & abcd & 4.9 & $\mathrm{ab}$ \\
\hline Sulfentrazone + imazethapyr & $140+37.5$ & 99 & abc & 100 & a & 0.9 & abc & 0.1 & a \\
\hline Sulfentrazone + imazethapyr & $210+37.5$ & 97 & abcd & 100 & a & 1.3 & $\mathrm{abc}$ & 0.4 & a \\
\hline $\begin{array}{l}\text { Sulfentrazone }+ \text { s-metolachlor }+ \\
\text { imazethapyr }\end{array}$ & $140+1050+37.5$ & 100 & $a b$ & 100 & a & 0.1 & a & 0.0 & a \\
\hline $\begin{array}{l}\text { Sulfentrazone }+ \text { s-metolachlor }+ \\
\text { imazethapyr }\end{array}$ & $210+1050+37.5$ & 100 & $a b$ & 100 & a & 0.6 & $\mathrm{ab}$ & 0.1 & a \\
\hline $\mathrm{SE}( \pm)$ & & & & & & & & & \\
\hline
\end{tabular}

${ }^{a}$ Abbreviations: PRE, pre-emergence; WAA, weeks after application. Means followed by the same letter within a column are not statistically different at $\alpha=0.05$ using Fisher's Protected LSD. Data are averaged for years and locations.

from the weedy control, and reduction was not improved when tank-mixed with s-metolachlor but was improved to almost $100 \%$ when tank-mixed with imazethapyr. Sulfentrazone (140 and 210 g·ai·ha ${ }^{-1}$ ) + s-metolachlor + imazethapyr reduced wild mustard density by $98 \%$ to $100 \%$ and biomass by $100 \%$.

\subsubsection{Barnyard Grass}

At 4 WAA, sulfentrazone (140 and $210 \mathrm{~g} \cdot a \mathrm{i} \cdot \mathrm{ha}^{-1}$ ) provided up to $81 \%$ control of barnyard grass (Table 6). Wilson et al. [14] also noted that sulfentrazone at these rates provided up to $78 \%$ control of barnyard grass. S-metolachlor provided $100 \%$ control and imazethapyr alone controlled barnyard grass by $69 \%$. Sulfentrazone (140 and $210 \mathrm{~g} \cdot \mathrm{ai}^{\mathrm{i}} \mathrm{ha}^{-1}$ ) $+\mathrm{s}$-metolachlor provided excellent control of barnyard grass, providing $100 \%$ control at 4 WAA and $98 \%$ to $100 \%$ control 8 WAA. Sulfentrazone (140 and $\left.210 \mathrm{~g} \cdot \mathrm{ai}^{-h^{-1}}{ }^{-1}\right)+$ imazethapyr had good (86\% to 92\%) control of barnyard grass at 4 WAA, but control decreased to $\leq 61 \%$ at 8 WAA. The co-application of sulfentrazone (140 and $\left.210 \mathrm{~g} \cdot \mathrm{ai}^{-h^{-1}}{ }^{-1}\right)+\mathrm{s}$-metolachlor + imazethapyr provided $100 \%$ control at 4 WAA and up to $98 \%$ control 8 WAA. There was no improvement in barnyard grass control at either 4 or 8 WAA when sulfentrazone was used in a co-application versus sulfentrazone applied alone. Sulfentrazone (140 and $210 \mathrm{~g} \cdot \mathrm{ai}^{\mathrm{h}} \mathrm{ha}^{-1}$ ), imazethapyr, and sulfentrazone + imazethapyr did not reduce barnyard grass density or biomass relative to the weedy control. In contrast, s-metolachlor reduced density and biomass by $97 \%$, and sulfentrazone + s-metolachlor reduced density and biomass by $98 \%$. Sulfentrazone $\left(140 \mathrm{~g} \cdot \mathrm{ai} \cdot \mathrm{ha} \mathrm{H}^{-1}\right)+\mathrm{s}$-metolachlor + imazethapyr reduced density and biomass by $100 \%$ and sulfentrazone $\left(210 \mathrm{~g} \cdot \mathrm{ai}^{\mathrm{h}} \mathrm{ha} \mathrm{g}^{-1}\right)+\mathrm{s}$-metolachlor + imazethapyr reduced density and biomass by $98 \%$.

\subsubsection{Green Foxtail}

At 4 WAA, sulfentrazone (140 and $210 \mathrm{~g} \cdot a \cdot \mathrm{ha}^{-1}$ ), s-metolachlor, and imazethapyr provided $80 \%, 90 \%$, 99\% and $85 \%$ control of green foxtail, respectively (Table 7). This is consistent with other studies, which found that sulfentrazone (260 g.ai-ha ${ }^{-1}$ ) provided $87 \%$ control of giant foxtail (Setaria faberi Herrm.) [18] and imazethapyr (30 g.ai ha ${ }^{-1}$ ) provided $75 \%$ to $93 \%$ control of green foxtail (Sikkema et al. 2005). At 8 WAA, control with sulfentrazone (140 and 210 g.ai ha ${ }^{-1}$ ) decreased to $62 \%$ and $79 \%$, respectively, control with s-metolachlor was 
Table 6. Mean control, plant density and biomass ratings for barnyard grass (Echinochloa crusgalli) treated with tank mixes of sulfentrazone, imazethapyr and s-metolachlor applied PRE in Ridgetown, ON and Exeter, ON, Canada over two years $(2014,2015)^{\mathrm{a}}$.

\begin{tabular}{|c|c|c|c|c|c|c|c|c|c|}
\hline \multirow{3}{*}{ Treatment } & Rate & \multicolumn{4}{|c|}{ Control } & \multicolumn{2}{|c|}{ Density 8 WAA } & \multicolumn{2}{|c|}{ Biomass 8 WAA } \\
\hline & & \multicolumn{2}{|c|}{4 WAA } & \multicolumn{2}{|c|}{8 WAA } & & & & \\
\hline & \multirow[t]{2}{*}{ g.ai ha ${ }^{-1}$} & \multicolumn{4}{|c|}{$\%$} & \multicolumn{2}{|c|}{ plants $\mathrm{m}^{-2}$} & \multicolumn{2}{|c|}{$\mathrm{g} \cdot \mathrm{m}^{-2}$} \\
\hline Untreated Control & & 0 & $\mathrm{~b}$ & 0 & e & 6.3 & c & 6.2 & $\mathrm{~b}$ \\
\hline Weed Free Control & & 100 & a & 100 & $\mathrm{a}$ & 0.0 & a & 0.0 & a \\
\hline S-metolachlor & 1050 & 100 & a & 99 & $\mathrm{ab}$ & 0.2 & $\mathrm{ab}$ & 0.2 & a \\
\hline Sulfentrazone & 140 & 81 & a & 86 & abcd & 3.4 & $\mathrm{abc}$ & 2.8 & $\mathrm{ab}$ \\
\hline Sulfentrazone & 210 & 74 & a & 73 & abcd & 4.7 & bc & 2.8 & $\mathrm{ab}$ \\
\hline Imazethapyr & 37.5 & 69 & a & 49 & d & 3.4 & $\mathrm{abc}$ & 2.6 & $\mathrm{ab}$ \\
\hline Sulfentrazone + s-metolachlor & $140+1050$ & 100 & $\mathrm{a}$ & 100 & a & 0.1 & a & 0.1 & a \\
\hline Sulfentrazone + s-metolachlor & $210+1050$ & 100 & $\mathrm{a}$ & 98 & abc & 0.1 & a & 0.1 & a \\
\hline Imazethapyr + s-metolachlor & $37.5+1050$ & 99 & a & 99 & $a b$ & 0.0 & a & 0.0 & a \\
\hline Sulfentrazone + imazethapyr & $140+37.5$ & 86 & a & 61 & bcd & 1.5 & abc & 1.4 & $\mathrm{ab}$ \\
\hline Sulfentrazone + imazethapyr & $210+37.5$ & 92 & a & 53 & $\mathrm{~cd}$ & 2.2 & abc & 1.8 & $\mathrm{ab}$ \\
\hline $\begin{array}{l}\text { Sulfentrazone }+ \text { s-metolachlor }+ \\
\text { imazethapyr }\end{array}$ & $140+1050+37.5$ & 100 & a & 96 & abcd & 0.0 & a & 0.0 & a \\
\hline $\begin{array}{l}\text { Sulfentrazone + s-metolachlor }+ \\
\text { imazethapyr }\end{array}$ & $210+1050+37.5$ & 100 & a & 98 & $\mathrm{ab}$ & 0.1 & a & 0.1 & a \\
\hline $\mathrm{SE}( \pm)$ & & \multicolumn{2}{|c|}{0.15} & \multicolumn{2}{|c|}{0.14} & \multicolumn{2}{|c|}{0.36} & \multicolumn{2}{|c|}{0.33} \\
\hline
\end{tabular}

${ }^{a}$ Abbreviations: PRE, pre-emergence; WAA, weeks after application. Means followed by the same letter within a column are not statistically different at $\alpha=0.05$ using Fisher's Protected LSD. Data are averaged for years and locations.

Table 7. Mean control, plant density and biomass ratings for green foxtail (Setaria viridis) treated with tank mixes of sulfentrazone, imazethapyr and s-metolachlor applied PRE in Ridgetown, ON and Exeter, ON, Canada over two years (2014, 2015) .

\begin{tabular}{|c|c|c|c|c|c|c|c|c|c|}
\hline \multirow{3}{*}{ Treatment } & Rate & \multicolumn{4}{|c|}{ Control } & \multicolumn{2}{|c|}{ Density 8 WAA } & \multicolumn{2}{|c|}{ Biomass 8 WAA } \\
\hline & & \multicolumn{2}{|c|}{4 WAA } & \multicolumn{2}{|c|}{8 WAA } & & & & \\
\hline & \multirow[t]{2}{*}{ g.ai ha ${ }^{-1}$} & 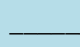 & 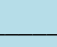 & 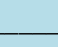 & - & \multicolumn{2}{|c|}{ plants $\mathrm{m}^{-2}$} & \multicolumn{2}{|c|}{$\mathrm{g} \cdot \mathrm{m}^{-2}$} \\
\hline Untreated Control & & 0 & g & 0 & $\mathrm{~d}$ & 73.6 & d & 40.3 & d \\
\hline Weed Free Control & & 100 & a & 100 & a & 0.0 & $\mathrm{a}$ & 0.0 & a \\
\hline S-metolachlor & 1050 & 99 & abc & 99 & a & 3.2 & abc & 1.0 & $a b$ \\
\hline Sulfentrazone & 140 & 80 & $\mathrm{f}$ & 62 & c & 17.3 & cd & 10.0 & c \\
\hline Sulfentrazone & 210 & 90 & def & 79 & bc & 14.8 & cd & 6.1 & c \\
\hline Imazethapyr & 37.5 & 85 & ef & 92 & $\mathrm{ab}$ & 28.7 & d & 4.1 & bc \\
\hline Sulfentrazone + s-metolachlor & $140+1050$ & 100 & abc & 99 & $\mathrm{a}$ & 0.8 & $\mathrm{a}$ & 0.7 & $a b$ \\
\hline Sulfentrazone + s-metolachlor & $210+1050$ & 99 & abc & 99 & a & 1.1 & $\mathrm{ab}$ & 0.5 & $a b$ \\
\hline Imazethapyr + s-metolachlor & $37.5+1050$ & 99 & abc & 99 & a & 2.4 & abc & 0.7 & $a b$ \\
\hline Sulfentrazone + imazethapyr & $140+37.5$ & 94 & cde & 95 & $\mathrm{ab}$ & 17.1 & cd & 2.2 & abc \\
\hline Sulfentrazone + imazethapyr & $210+37.5$ & 96 & bcd & 97 & $\mathrm{ab}$ & 13.0 & bcd & 2.2 & abc \\
\hline $\begin{array}{l}\text { Sulfentrazone }+ \text { s-metolachlor }+ \\
\text { imazethapyr }\end{array}$ & $140+1050+37.5$ & 99 & abc & 100 & a & 1.0 & a & 0.2 & a \\
\hline $\begin{array}{l}\text { Sulfentrazone + s-metolachlor + } \\
\text { imazethapyr }\end{array}$ & $210+1050+37.5$ & 100 & ab & 100 & a & 0.7 & a & 0.2 & a \\
\hline $\mathrm{SE}( \pm)$ & & & & & & & & & \\
\hline
\end{tabular}

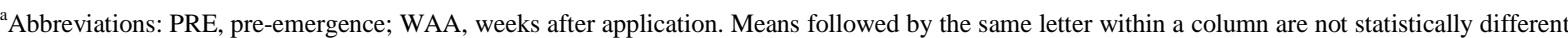
at $\alpha=0.05$ using Fisher's Protected LSD. Data are averaged for years and locations. 
stable, and control with imazethapyr increased to $92 \%$. Sulfentrazone + s-metolachlor provided $99 \%$ to $100 \%$ control 4 and 8 WAA. This was an improvement in control compared to sulfentrazone alone, but not compared to s-metolachlor alone. The co-application of sulfentrazone $+\mathrm{s}$-metolachlor reduced green foxtail density by 99\% and biomass up to $99 \%$. Sulfentrazone $\left(140 \mathrm{~g} \cdot \mathrm{ai} \cdot \mathrm{ha}^{-1}\right)+$ imazethapyr provided $94 \%$ control at 4 WAA, which was an improvement compared to sulfentrazone on its own, while sulfentrazone $\left(210 \mathrm{~g} \cdot \mathrm{ai} \cdot \mathrm{ha}^{-1}\right)+$ imazethapyr provided $96 \%$ control but did not improve control compared to sulfentrazone. At 8 WAA, sulfentrazone (140 and $210 \mathrm{~g} \cdot \mathrm{ai} \cdot \mathrm{ha}^{-1}$ ) + imazethapyr provided 95\% to 97\% control, which was greater than sulfentrazone (140 $\mathrm{g} \cdot \mathrm{ai} \cdot \mathrm{ha}^{-1}$ ) alone but not sulfentrazone $\left(210 \mathrm{~g} \cdot \mathrm{ai} \cdot \mathrm{ha}^{-1}\right)$. Sulfentrazone + imazethapyr reduced foxtail biomass by $95 \%$, but did not reduce foxtail density relative to the weedy control. Sulfentrazone (140 and $\left.210 \mathrm{~g} \cdot \mathrm{ai} \cdot \mathrm{ha}^{-1}\right)+$ s-metolachlor + imazethapyr provided $99 \%$ to $100 \%$ control at 4 and 8 WAA, and decreased density by $99 \%$ and biomass by $100 \%$.

\subsection{Seed Moisture Content and Yield}

There was no impact on white bean seed moisture at harvest, indicating that weed interference and/or herbicide injury did not cause delayed maturity (Table 1). However, there were variations in yield amongst the treatments. Weed interference reduced white bean yield 52\%. Weed interference with sulfentrazone alone at 140 and 210 $\mathrm{g} \cdot \mathrm{ai} \cdot \mathrm{ha}^{-1}$ resulted in the lowest numeric yields among the herbicide treatments evaluated at 1.42 and $1.49 \mathrm{~T} \cdot \mathrm{ha}^{-1}$, respectively. Reduced weed interference with s-metolachlor, imazethapyr, and imazethapyr + s-metolachlor produced yields that were $33 \%, 34 \%$ and $32 \%$ lower than the weed-free control, and were not different than the weedy control. The addition of s-metolachlor or imazethapyr to sulfentrazone did not improve yields compared to sulfentrazone alone, and were not improved relative to the weedy control. Sulfentrazone $\left(210 \mathrm{~g} \cdot \mathrm{ai}^{\mathrm{i}} \mathrm{ha}^{-1}\right)+$ s-metolachlor + imazethapyr produced the third lowest yield at $1.60 \mathrm{~T} \cdot \mathrm{ha}^{-1}$. As this co-application produced the highest amount of injury, it is likely this $37 \%$ yield loss is mostly attributed to herbicide injury rather than weed interference. In contrast, sulfentrazone $\left(140 \mathrm{~g} \cdot \mathrm{ai} \cdot \mathrm{ha}^{-1}\right)+\mathrm{s}$-metolachlor + imazethapyr produced the highest yield of all the herbicide treatments at $1.89 \mathrm{~T} \cdot \mathrm{ha}^{-1}$, and was the only treatment that produced a yield greater than the weedy control. It is likely that the high yield produced by this co-application is due to less injury and better broad spectrum weed control compared to the other tank mixes evaluated in this study.

\section{Conclusion}

This study found that PRE applications of sulfentrazone provide excellent control of pigweeds and common lambsquarters. The addition of imazethapyr to sulfentrazone increased wild mustard control to $100 \%$, and while it did improve common ragweed control compared to sulfentrazone alone, it did not improve control to an acceptable level. Sulfentrazone + s-metolachlor + imazethapyr provided excellent control of pigweeds, lambsquarters, wild mustard, barnyard grass and green foxtail, but did not have an acceptable margin of crop safety. Based on this study, tank mixes of sulfentrazone + s-metolachlor + imazethapyr are too injurious to Ontario white bean for registration. Further research is needed to determine if sulfentrazone is a suitable herbicide for weed management programs in other market classes of dry beans in Ontario.

\section{Acknowledgements}

The authors would like to acknowledge Todd Cowan for his expertise and technical assistance with these studies. Funding for this project was provided in part by Ontario Bean Growers (OBG) and the GF program of the Agricultural Adaptation Council.

\section{References}

[1] Kulasekera, K. (2015) Estimated Area, Yield, Production and Farm Value of Specified Field Crops, Ontario, 2011-2015. Ontario Ministry of Agriculture, Food and Rural Affairs. http://www.omafra.gov.on.ca/english/stats/crops/estimate_new.htm\#metric

[2] Statistics Canada (2015) Table 001-0010_Estimated Areas, Yield, Production and Average Farm Price of Principal Field Crops, in Metric Units, Annual, CANSIM (Database). http://www5.statcan.gc.ca/cansim/a33?RT=TABLE\&themeID=3953\&spMode=tables\&lang=eng

[3] Malik, V.S., Swanton, C.J. and Michaels, T.E. (1993) Interaction of White Bean (Phaseolus vulgaris) Market Class, 
Row Spacing, and Seeding Density with Annual Weeds. Weed Sci., 41, 62-68.

[4] Soltani, N., Nurse, R.E., Shropshire, C. and Sikkema, P.H. (2014) Weed Control in White Bean with Various Halosulfuron Tankmixes. Advances in Agriculture, 2014, Article ID: 391634.

[5] Soltani, N., Nurse, R.E., Shropshire, C. and Sikkema, P.H. (2014). Weed Control with Halosulfuron Applied Preplant Incorporated, Preemergence or Postemergence in White Bean. Agricultural Sciences, 5, 875-881. http://dx.doi.org/10.4236/as.2014.510094

[6] Anonymous (2012) Authority ${ }^{\circledR} 480$ Herbicide Product Label. FMC Corporation, 4 p.

[7] Niekamp, J.W., Johnson, W.G. and Smeda, R.J. (1999) Broadleaf Weed Control with Sulfentrazone and Flumioxazin in No-Tillage Soybean (Glycine max). Weed Technology, 13, 233-238.

[8] Niekamp, J.W. and Johnson, W.G. (2001) Weed Management with Sulfentrazone and Flumioxazin in No-Tillage Soyabean (Glycine max). Crop Protection, 20, 215-220. http://dx.doi.org/10.1016/S0261-2194(00)00129-0

[9] Shaner, D.L. (2014) Herbicide Handbook. 10th Edition, Weed Science Society of America, Lawrence, 513 p.

[10] Ontario Ministry of Agriculture, Food and Rural Affairs (2014) Guide to Weed Control. Publication 75, Toronto, 2014, 1-416.

[11] Sikkema, P.H., Deen, W. and Vyas, S. (2005) Weed Control in Pea with Reduced Rates of Imazethapyr Applied Preemergence and Postemergence. Weed Technology, 19, 14-18. http://dx.doi.org/10.1614/WT-03-051R3

[12] Soltani, N., Van Eerd, L., Vyn, R.J., Shropshire, C. and Sikkema, P.H. (2007) Weed Control, Environmental Impact and Profitability of Reduced Rates of Imazethapyr in Combination with Dimethenamid in Dry Beans. Canadian Journal of Plant Science, 87, 671-678. http://dx.doi.org/10.4141/CJPS06014

[13] Soltani, N., Shropshire, C. and Sikkema, P.H. (2014) Response of Dry Bean to Sulfentrazone plus Imazethapyr. International Journal of Agronomy, 1-6.

[14] Walsh, K.D., Soltani, N., Hooker, D.C., Nurse, R.E. and Sikkema, P.H. (2015) Biologically Effective Rate of Sulfentrazone Applied Pre-Emergence in Soybean. Canadian Journal of Plant Science, 95, 1-6. http://dx.doi.org/10.4141/cjps-2014-264

[15] Wilson, D.E., Nissen, S.J. and Thompson, A. (2002) Potato (Solanum tuberosum) Variety and Weed Response to Sulfentrazone and Flumioxazin. Weed Technology, 16, 567-574. http://dx.doi.org/10.1614/0890-037X(2002)016[0567:PSTVAW]2.0.CO;2

[16] Bailey, W.A., Wilson, H.P. and Hines, T.E. (2002) Response of Potato (Solanum tuberosum) and Selected Weeds to Sulfentrazone. Weed Technology, 16, 651-658. http://dx.doi.org/10.1614/0890-037X(2002)016[0651:ROPSTA]2.0.CO;2

[17] Brown, D. and Masiunus, J. (2002) Evaluation of Herbicides for Pumpkin (Curcurbita spp.). Weed Technology, 16, 282-292. http://dx.doi.org/10.1614/0890-037X(2002)016[0282:EOHFPC]2.0.CO;2

[18] Krausz, R.F., Kapusta, G. and Matthews, J.L. (1998) Sulfentrazone for Weed Control in Soybean (Glycine max). Weed Technology, 12, 684-689.

\section{Submit or recommend next manuscript to SCIRP and we will provide best service for you:}

Accepting pre-submission inquiries through Email, Facebook, LinkedIn, Twitter, etc.

A wide selection of journals (inclusive of 9 subjects, more than 200 journals)

Providing 24-hour high-quality service

User-friendly online submission system

Fair and swift peer-review system

Efficient typesetting and proofreading procedure

Display of the result of downloads and visits, as well as the number of cited articles

Maximum dissemination of your research work

Submit your manuscript at: http://papersubmission.scirp.org/ 